\title{
DESIGUALDADE ESTRUTURAL DISCRETA DE MEMBROS INFERIORES É SUFICIENTE PARA CAUSAR ALTERAÇÃO CINÉTICA NA MARCHA DE CORREDORES?
}

\author{
IS STRUCTURAL AND MILD LEG LENGTH DISCREPANCY ENOUGH TO CAUSE A KINETIC CHANGE IN \\ RUNNERS' GAIT?
}

\author{
Carla Sonsino Pereira ${ }^{1}$, Isabel de Camargo Neves Sacco ${ }^{2}$
}

\begin{abstract}
RESUMO
Desigualdade de membros inferiores (DMI) está presente em cerca de $70 \%$ da população geral, podendo ser do tipo estrutural onde existe diferença no comprimento de estruturas ósseas, ou funcional, como resultado de alterações mecânicas dos membros inferiores. A desigualdade pode ainda ser classificada quanto a sua magnitude, sendo discreta, moderada, ou grave. As desigualdades discretas têm sido associadas especificamente à fratura por estresse, dor lombar e osteoartrite, e quando uma desigualdade está presente em indivíduos cuja sobrecarga mecânica é acentuada pela sua prática profissional, diária ou recreativa, estas alterações ortopédicas podem se manifestar precoce e gravemente. O objetivo deste estudo foi analisar e comparar a força reação do solo (FRS) durante a marcha de corredores com e sem DMl discreta. Os resultados mostraram que os sujeitos com desigualdades de 0,5 a 2,0 cm apresentaram no membro menor maiores valores da força vertical mínima $(0,57 \pm 0,07 \mathrm{PC})$ em relação ao membro maior $(0,56 \pm$ 0,08 PC). Logo, sujeitos com DMl discreta adotam mecanismos compensatórios capazes de gerar sobrecarga adicional ao sistema musculoesquelético para promover uma marcha simétrica como demonstrado pelos valores do Índice de Simetria Absoluto das variáveis da FRS vertical e horizontal.
\end{abstract}

Descritores: Biomecânica; Marcha; Desigualdade de membros inferiores.

Citação: Pereira CS, Sacco ICN. Desigualdade estrutural discreta de membros inferiores é suficiente para causar alteração cinética na marcha de corredores? Acta Ortop Bras. [periódico na Internet]. 2008; 16(1):29-31. Disponível em URL: http://www.scielo.br/aob.

\section{INTRODUÇÃO}

Desigualdade de membros inferiores (DMI) pequenas ou discretas, isto é, inferiores a 3,0 cm, têm sido consideradas suficientes para causar alterações ortopédicas como dor lombar, fratura por stress e osteoartrite nas articulações dos membros inferiores (MMII)(1,2). Além da classificação pela sua magnitude, as desigualdades ainda podem ser classificadas quanto a sua etiologia, sendo estrutural quando existe diferença no comprimento de estruturas ósseas ${ }^{(3)}$; ou funcional como resultado de alterações mecânicas do membro inferior, e são observadas em 65 a 70\% da população saudável(4,5). São vários os métodos clínicos e radiográficos utilizados para quantificar esta desigualdade, mas recentemente a análise biomecânica da marcha tem se mostrado eficiente em detectar assimetrias entre os MMII uma vez que alterações na dinâmica da marcha de sujeitos com DMI têm sido descritas pela literatura ${ }^{(4,6-9)}$. Entretanto, dentro do nosso conhecimento, não há um consenso na literatura se as desigualdades discretas são suficientes ou não para causar alguma alteração biomecânica ou assimetria na marcha.

\section{SUMMARY}

Leg length discrepancy (LLD) affects about $70 \%$ of the general population, and can be either structural - when the difference occurs in bone structures - or functional, because of mechanical changes at the lower limbs. The discrepancy can be also classified by its magnitude into mild, intermediate, or severe. Mild LLD has been particularly associated with stress fracture, low back pain and osteoarthritis, and when the discrepancy occurs in subjects whose mechanical loads are increased by their professional, daily or recreational activities, these orthopaedic changes may appear early and severely. The aim of this study was to analyze and compare ground reaction force (GRF) during gait in runners with and without mild LLD. Results showed that subjects with mild LLD of 0.5 to $2.0 \mathrm{~cm}$ presented higher values of minimum vertical GRF $(0.57 \pm 0.07 \mathrm{BW})$ at the shorter limb compared to the longer limb $(0.56 \pm 0.08 \mathrm{BW})$ Therefore, subjects with mild LLD adopt compensatory mechanisms that cause additional overloads to the musculoskeletal system in order to promote a symmetrical gait pattern as showed by the values of absolute symmetric index of vertical and horizontal GRF variables.

Keywords: Biomechanics; Gait; Leg Length Inequality.

Citation: Pereira CS, Sacco ICN. Is structural and mild leg length discrepancy enough to cause a kinetic change in runners. gait? Acta Ortop Bras. [serial on the Internet]. 2008; 16(1):29-31. Available from URL: http://www.scielo.br/aob.

Brunet et a/(1), após analisar 1505 questionários respondidos por corredores, detectaram que os desequilíbrios biomecânicos, como a DMI, parecem ser um dos maiores contribuintes para a ocorrência de lesões na corrida, e que essas lesões podem ser explicadas pela sobrecarga sofrida pelos MMII, que pode atingir de duas a quatro vezes o peso corporal para corridas recreativas e de velocidade respectivamente ${ }^{(10)}$.

Kaufman et a/(4) após analisar a marcha de crianças com DMI atestaram que desigualdades superiores a 2,0 cm resultam em assimetria de marcha se comparado com crianças sem desigualdade. Song et $\mathrm{a}^{\left({ }^{(7)}\right.}$ após avaliar a cinética e a cinemática da marcha de adolescentes, não observaram mecanismos compensatórios associados às desigualdades de 2,2 $\pm 4,5 \%$ do membro inferior maior, e ainda, que os sujeitos com tais desigualdades não demonstraram nenhuma assimetria cinemática ou cinética da marcha. Liu et a/(8) ao estudar 30 jovens com idade média de 14 anos, mostraram que desigualdades de até $2,33 \mathrm{~cm}$ levavam a uma marcha simétrica normal, e concluíram que pacientes com DMI adotam mecanis-

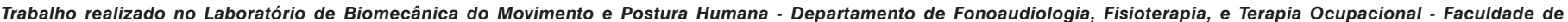
Medicina da Universidade de São Paulo - FMUSP.

Endereço para correspondência: Rua Cipotânea, 51 - Cidade Universitária - São Paulo - SP - Cep: 05360-160 - E-mail: carlaspd@usp.br

1 Pós-Graduanda em Ciências da Reabilitação do Departamento de Fonoaudiologia, Fisioterapia e Terapia Ocupacional - Faculdade de Medicina da Universidade de São Paulo - FMUSP. 2 Professora, Doutora do Departamento de Fonoaudiologia, Fisioterapia e Terapia Ocupacional - Faculdade de Medicina - USP. 
mos compensatórios, tais como: aumento da flexão do Ml longo, aumento da extensão do Ml curto, pronação do pé no Ml longo, supinação do pé no Ml curto, entre outros na tentativa de corrigir a desigualdade ${ }^{(7,11)}$, já White et a/(9) após comparar a marcha de adultos com e sem desigualdade discreta, e desigualdade simulada, encontraram que desigualdades maiores que $1,0 \mathrm{~cm}$ resultam em assimetrias de cargas baseados nos valores do Índice de Simetria da força reação do solo (FRS) vertical.

Em função das controvérsias encontradas na literatura e por acreditar que desigualdades discretas já são capazes de causar algum tipo de alteração nos padrões dinâmicos da locomoção e cronicamente nas estruturas do aparelho locomotor, é de extrema importância analisar os efeitos dessas desigualdades discretas em sujeitos praticantes de corrida, uma vez que estes sofrem alta sobrecarga em seu aparelho locomotor devido a rotina cíclica e intensa de treinamento. Diante disso, o objetivo deste estudo foi verificar se corredores de média e longa distância com DMI, do tipo estrutural e discreta maior que 0,5 cm, apresentam alterações nas variáveis da força reação do solo horizontal e vertical durante a marcha; e ainda, se apresentam alterações nas mesmas variáveis quando comparados à corredores sem a desigualdade.

\section{MATERIAL E MÉTODOS}

Quarenta e sete corredores de média e longa distância, de ambos os sexos, voluntários, assintomáticos, praticantes de corrida pelo menos três vezes por semana por pelo menos um ano, com idades entre 18 e 45 anos foram avaliados neste estudo prospectivo. Todos os sujeitos foram submetidos a um questionário e o comprimento de seus MMII foi aferido por meio de uma medida radiográfica - a escanometria - realizadas por um único técnico em radiologia. Os sujeitos deitaram em supino na mesa de raios-X mantendo a pelve nivelada e os MMII em posição anatômica. Uma régua metálica rádio-opaca foi colocada na mesa entre os MMII dos sujeitos, e uma série de três radiografias foi feita, com a ampola de raios- $X$ sendo focada seqüencialmente nas articulações dos quadris, joelhos e tornozelos, conforme descrito por Cunha et al ${ }^{(12)}$ e Terry et al ${ }^{(13)}$. Todos os sujeitos foram convidados a realizar a análise biomecânica da marcha, entretanto 13 sujeitos desistiram de continuar no estudo. Os 34 sujeitos restantes consentiram sua participação por meio de um termo informado aprovado pela Comissão de Ética para Análise de Projetos de Pesquisa da instituição local. Estes foram divididos em 2 grupos, desigualdade $(n=22)$ com desigualdade estrutural $\geq 0,5$ $\mathrm{cm}$ e controle $(\mathrm{n}=12) \mathrm{com}$ desigualdade estrutural $<0,5 \mathrm{~cm}^{(14)}$.

$\mathrm{Na}$ escanometria de membro inferior, o comprimento do fêmur e da tíbia foram definidos conforme descrito por Cunha et $\mathrm{al}^{\left({ }^{(12)} \text {. }\right.}$ A medida do comprimento dos MMII foi obtida pela soma dos comprimentos do fêmur e da tíbia, sendo expressa em centímetros, e a DMI foi obtida pela diferença absoluta em centímetros do comprimento do membro inferior maior $\left(\mathrm{MI}_{\text {mair }}\right)$ pelo comprimento do membro inferior menor $\left(\mathrm{MI}_{\text {menor }}\right)$. A partir deste valor pôde-se expressar a desigualdade normalizada entre os MMII por meio do percentual do comprimento da extremidade maior4, definida pela equação 1:

$$
\text { Desigualdade normalizada }(\%)=\frac{M I_{\text {Maior }}-M I_{\text {Menor }}}{M I_{\text {Maior }}(\mathrm{cm})} \text { (1) }
$$

Optou-se por utilizar também a desigualdade normalizada nas análises por acreditar que quanto maior for a estatura do paciente mais bem tolerada será a DMI ${ }^{(15)}$.

As variáveis da força reação do solo (FRS) componente vertical e horizontal foram mensuradas durante a marcha por meio de uma plataforma de força, tipo AMTI - modelo OR 62000, embutida no centro de uma passarela de 10 metros de comprimento. Cinco passadas intermediárias foram coletadas para o $\mathrm{MI}_{\text {maior }}$ e cinco para o $\mathrm{MI}_{\text {menor }}$ Durante as coletas, os sujeitos caminharam em cadência auto-selecionada e fizeram uso do tênis que utilizam durante a prática da corrida como forma de diminuir o efeito retroativo.
Antes de iniciar a aquisição dos dados, os sujeitos foram orientados a andar pela passarela em uma velocidade confortável quantas vezes fosse necessário até que se sentissem habituados ao ambiente de coleta, no entanto só se iniciava à aquisição dos dados propriamente dita quando um padrão similar na curva de FRS vertical, isto é, um impulso semelhante de desaceleração e aceleração durante o contato do pé com o solo, fosse observado em duas tentativas seqüenciais ${ }^{(16)}$.

Os dados foram adquiridos com uma freqüência de amostragem de $1000 \mathrm{~Hz}$, e um filtro passa baixa de $100 \mathrm{~Hz}$ foi utilizado durante o tratamento matemático dos dados respeitando os resultados obtidos pelo filtro FFT realizado ${ }^{(17)}$. Os dados foram ainda normalizados pelo peso corporal (PC) de cada sujeito.

As variáveis da FRS componente vertical analisadas foram (Figura 1): primeiro pico de força $\left(F z_{1}\right)$; força mínima $\left(F z_{\text {min }}\right)$; segundo pico de força $\left(F z_{2}\right)$; a taxa de crescimento $1\left(T C z_{1}=F z_{1} / \Delta t z_{1}\right)$; a taxa de crescimento $2\left(\mathrm{TCz}_{2}=\mathrm{Fz} / \Delta \mathrm{tz} \mathrm{z}_{2}\right)$; e a taxa de Push off (PO) ou taxa de descarga $\left[\mathrm{Fz}_{2} /\left(\mathrm{t}_{\text {tinal }}-\mathrm{tz}_{2}\right)\right]$. As variáveis da $\mathrm{FRS}$ horizontal analisadas foram (Figura 2): pico de força de desaceleração ( $\left.F x_{\text {min }}\right)$, pico de força de aceleração (Fx $\left.x_{\text {máx }}\right)$; Impulso de Desaceleração (ID); e Impulso de Aceleração (IA). Outra variável estudada foi o índice de simetria absoluto (ISA) das seguintes variáveis da $\mathrm{FRS}$ : $F z_{1}$; $\mathrm{Fz}_{\min } ; \mathrm{Fz}_{2} ; \mathrm{Fx}_{\max }$; e $\mathrm{Fx}_{\min }$. O valor do índice igual a zero indica que não há diferença entre as variáveis para o lado maior e o menor e, portanto, há uma perfeita simetria da marcha(18). Tal índice é obtido por meio da seguinte equação (2) (19):

$$
I S A(\%)=\frac{\mid X_{\text {Maior }}-X_{\text {Menor }} /}{1 / 2\left(X_{\text {Maior }}+X_{\text {Menor }}\right)} \cdot 100
$$

onde $\mathrm{X}_{\text {maior }}$ é a variável de marcha para o $\mathrm{Ml}$ maior, e $\mathrm{X}_{\text {menor }}$ é a variável correspondente para o MI menor.

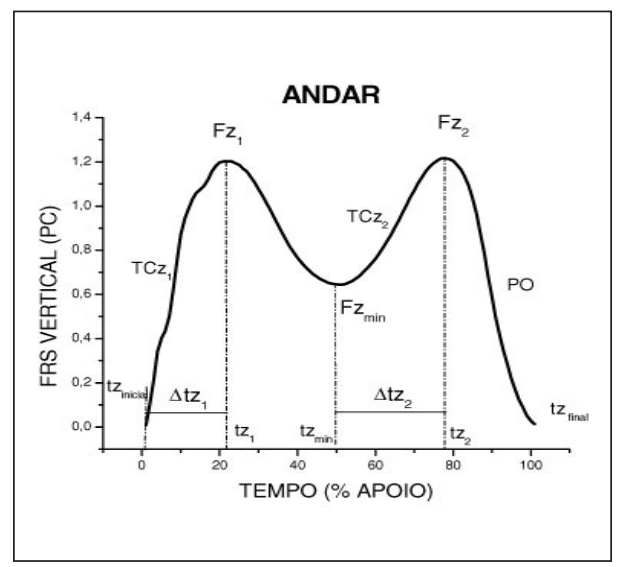

Figura 1 - Representação das variáveis estudadas da FRS vertical durante $o$ andar: $F z_{1}$, o primeiro pico de força; $F z_{\text {min }}$, força mínima; $F z_{2}$ o segundo pico de força; $T C z_{1}$ a taxa de crescimento 1 , $T \mathrm{Tz}_{2}$, a taxa de crescimento 2, e PO, taxa de push off.

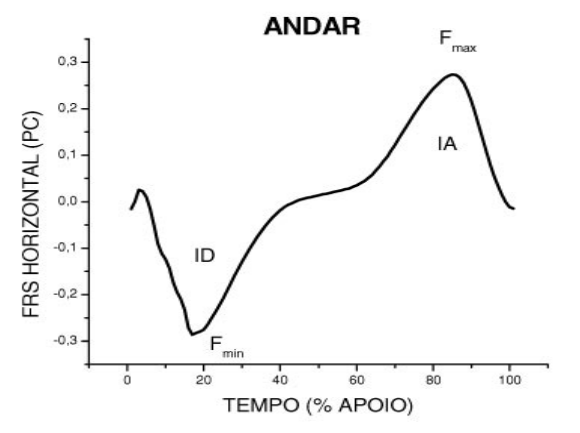

Figura 2 - Representação das variáveis estudadas da FRS horizontal durante o andar: $F x_{\min ,}$ força mínima; $F x_{\text {máx }}$ força máxima; impulso de desaceleração (ID); e impulso de aceleração (IA). 
O tratamento matemático dos dados e o cálculo das variáveis da FRS foram automatizados e realizados por meio de uma rotina matemática desenvolvida pelos pesquisadores em código Matlab. Já as análises estatísticas foram feitas no programa Statistica 6.0. Os dados foram representados descritivamente por meio de médias, desvios-padrão e freqüências. A normalidade dos dados da FRS foi testada por meio do teste de Shapiro Wilks e diante do padrão de distribuição normal apresentado, utilizou-se o teste $t$ para amostras independentes para as comparações intergrupos, e o teste $t$ pareado para as comparações intragrupos (entre o $\mathrm{MI}_{\text {mair }}$ e o $\mathrm{MI}_{\text {menor }}$ ). Adotou-se um $\alpha$ de $5 \%$ para diferenças estatisticamente significativas.

\section{RESULTADOS}

Dos 47 sujeitos avaliados (33 homens e 14 mulheres; idade média de 31,3 \pm 5,3 anos), somente 10,6\% (5) afirmaram ter conhecimento da DMI, e 8,5\% dos sujeitos (4) usavam ou já usaram algum tipo de órtese nos calçados. A desigualdade média absoluta entre os MMII apresentada por estes 47 sujeitos variou de zero a 2,25 cm. Cerca de $6 \%$ (3) dos sujeitos não apresentaram desigualdade estrutural entre os MMII, em 10,6\% (5) dos sujeitos a desigualdade foi devido à diferença de comprimento do fêmur, em 10,6\% (5) devido à diferença de comprimento na tíbia, e em 72,3\% (34) dos sujeitos a DMI foi devido a diferença de tamanho tanto do fêmur quanto da tíbia.

O grupo desigualdade (GD) foi composto por 16 homens e 6 mulheres; idade média de 30,6 \pm 3,9 anos; desigualdade média absoluta de 1,0 \pm 0,5 cm; e desigualdade média normalizada de $1,1 \pm 0,5 \%$; e o grupo controle (GC) foi composto por 8 homens e 4 mulheres; idade média de 30,8 \pm 5,4 anos; desigualdade média absoluta de 0,2 $\pm 0,2 \mathrm{~cm}$; e desigualdade média normalizada de $0,3 \pm 0,2 \%$.

Os resultados da análise biomecânica da marcha estão descritos na Tabela 1 e não foram observadas diferenças estatisticamente significativas nas variáveis da FRS vertical e horizontal nas comparações intergrupos, tanto no $\mathrm{MI}_{\text {maior }}$ quanto no $\mathrm{MI}_{\text {menor' }}$ As Figuras 3 e 4 ilustram a força reação do solo média vertical e horizontal para os grupos estudados.

Na comparação intragrupo, o GC não apresentou diferenças estatísticas entre os MMll em todas as variáveis, como esperado, e o $\mathrm{GD}$ apresentou a variável $\mathrm{Fz}$ min significativamente maior no $\mathrm{MI}_{\text {menor }}$ em relação ao maior $(p=0,0412)$.

Nenhum dos valores calculados do ISA para as variáveis avaliadas da FRS vertical e horizontal foi diferente estatisticamente entre GC e GD. E de um modo geral, observou-se que tanto em GC quanto em GD os valores do ISA permaneceram pequenos para as variáveis da FRS vertical, e muito próximos de zero para as variáveis da FRS horizontal como se pode observar na Tabela 2.
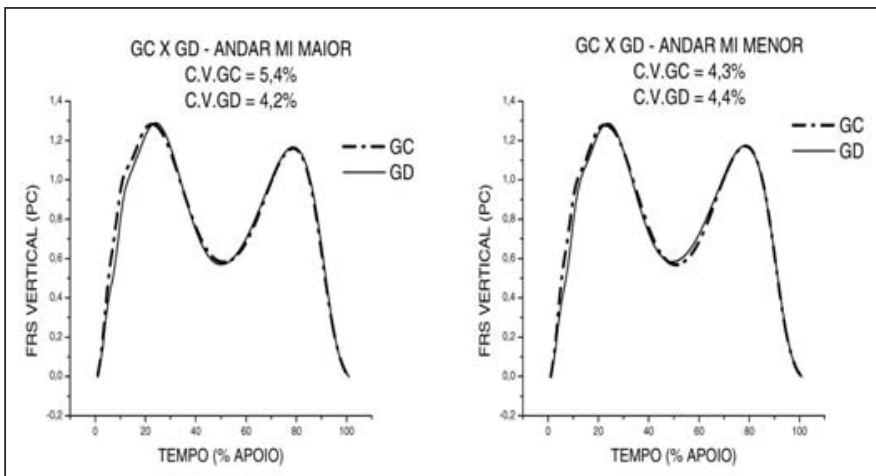

Figura 3: Curvas Médias da FRS vertical dos Grupos Controle (GC) e Desigualdade (GD), e CV (\%), lados maior e menor.
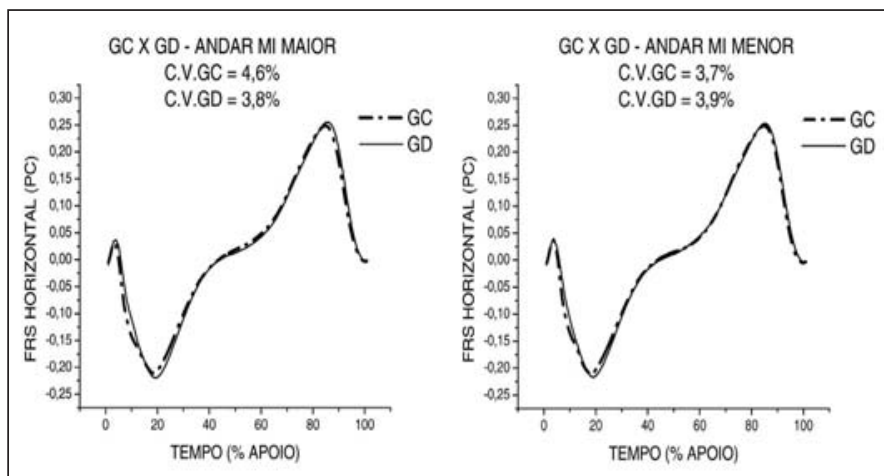

Figura 4: Curvas médias da FRS horizontal dos Grupos Controle (GC) e desigualdade $(G D)$, e CV (\%), lados maior e menor.

\section{DISCUSSÃO}

Mesmo na presença de desigualdade estrutural discreta de MMII, apenas quando se comparou os MMII de sujeitos com desigualdades maiores que 0,5 cm puderam-se observar alterações na FRS vertical durante o andar. E mesmo com esta presença de desigualdade e alteração da FRS, tanto os sujeitos do grupo controle como os sujeitos do grupo desigualdade apresentaram uma marcha simétrica de acordo com os valores do ISA calculados.

\begin{tabular}{|c|c|c|c|c|c|c|}
\hline \multirow[b]{2}{*}{ VARIÁVEIS } & \multicolumn{2}{|c|}{ GD (n=22) } & \multirow[b]{2}{*}{$p$} & \multicolumn{2}{|c|}{$\mathrm{GC}(\mathrm{n}=12)$} & \multirow[b]{2}{*}{$\mathbf{p}$} \\
\hline & MI Maior & MI Menor & & MI Maior & MI Menor & \\
\hline $\mathrm{Fz}_{1}(\mathrm{PC})$ & $1,30 \pm 0,09$ & $1,29 \pm 0,10$ & 0,5502 & $1,29 \pm 0,12$ & $1,29 \pm 0,10$ & 0,7336 \\
\hline $\mathrm{Fz}_{2}(\mathrm{PC})$ & $1,17 \pm 0,07$ & $1,18 \pm 0,06$ & 0,4415 & $1,16 \pm 0,08$ & $1,17 \pm 0,06$ & 0,2219 \\
\hline$F z_{\text {min }}(P C) *$ & $0,56 \pm 0,08$ * & $0,57 \pm 0,07^{\star}$ & 0,0412 & $0,57 \pm 0,10$ & $0,56 \pm 0,10$ & 0,1062 \\
\hline $\mathrm{TC}_{1}(\mathrm{PC} / \mathrm{s})$ & $9,66 \pm 1,79$ & $9,68 \pm 1,73$ & 0,9026 & $10,44 \pm 1,72$ & $10,42 \pm 1,62$ & 0,9323 \\
\hline $\mathrm{TC}_{2}(\mathrm{PC} / \mathrm{s})$ & $6,75 \pm 0,92$ & $6,73 \pm 0,86$ & 0,8885 & $7,04 \pm 0,86$ & $7,16 \pm 0,80$ & 0,4363 \\
\hline $\mathrm{PO}(\mathrm{PC} / \mathrm{s})$ & $8,70 \pm 1,33$ & $8,57 \pm 1,17$ & 0,3714 & $8,89 \pm 1,34$ & $8,89 \pm 1,41$ & 0,9917 \\
\hline$F x_{\max }(P C)$ & $0,26 \pm 0,03$ & $0,25 \pm 0,03$ & 0,6248 & $0,25 \pm 0,03$ & $0,25 \pm 0,03$ & 0,8455 \\
\hline$F x_{\min }(P C)$ & $0,22 \pm 0,04$ & $0,22 \pm 0,04$ & 0,4741 & $0,21 \pm 0,04$ & $0,21 \pm 0,03$ & 0,9128 \\
\hline $\operatorname{Imp} P_{\text {Desaceleração }}$ & $4,33 \pm 0,76$ & $4,22 \pm 0,96$ & 0,3991 & $4,39 \pm 1,02$ & $4,23 \pm 0,82$ & 0,2178 \\
\hline Imp ${ }_{\text {Aceleraçăo }}$ & $5,90 \pm 0,65$ & $5,87 \pm 0,70$ & 0,8135 & $5,89 \pm 0,81$ & $5,75 \pm 0,78$ & 0,1677 \\
\hline
\end{tabular}

Teste $t$ pareado, teste t para amostras independentes $(p<0,05)$. ${ }^{*}$ representa diferença estatisticamente significativa.

Tabela 1 - Valores das médias, desvio padrão e valores de $p$ das variáveis da FRS vertical e horizontal normalizadas pelo peso corporal (PC) durante o andar no Grupo Desigualdade (GD) e no Grupo controle (GC). 


\begin{tabular}{c|c|c|c}
\hline VARIÁVEIS & GD (n=22) & $\mathbf{G C}(\mathbf{n}=\mathbf{1 2})$ & $\mathbf{p}$ \\
\hline $\mathrm{Fz}_{1}(\%)$ & $3,5 \pm 1,8$ & $4,0 \pm 2,0$ & 0,4093 \\
\hline $\mathrm{Fz}_{\min }(\%)$ & $2,1 \pm 0,9$ & $1,9 \pm 0,9$ & 0,6362 \\
\hline $\mathrm{Fz}_{2}(\%)$ & $3,4 \pm 1,6$ & $3,9 \pm 1,5$ & 0,4141 \\
\hline $\mathrm{Fx}_{\max }(\%)$ & $0,6 \pm 0,4$ & $0,5 \pm 0,2$ & 0,2898 \\
\hline $\mathrm{Fx}_{\min }(\%)$ & $0,6 \pm 0,4$ & $0,6 \pm 0,3$ & 0,5710 \\
\hline
\end{tabular}

Teste t para amostras independentes $(p<0,05)$.

Tabela 2 - Valores das médias, desvio padrão e valores de $p$ dos Índices de Simetria Absolutos das variáveis de FRS vertical e horizontal no Grupo Desigualdade (GD) e no Grupo Controle (GC).

Este estudo demonstrou por meio da análise biomecânica da marcha, que desigualdades estruturais discretas de até $2,25 \mathrm{~cm}$ não foram suficientes para causar qualquer alteração na FRS horizontal e vertical em comparação aos sujeitos sem desigualdade. Mas ao se comparar os MMII maior e menor em função da desigualdade de 0,5 - 2,0 cm (intervalo de confiança de $99 \%$ ), o MI menor $_{\text {dos sujeitos }}$ com desigualdade apresentou maiores valores de $\mathrm{Fz}_{\text {min }}$, o que pode indicar uma menor eficiência mecânica deste membro devido a uma menor absorção de energia, uma vez que quanto menor o valor de $\mathrm{Fz}_{\min }$, maior será a absorção de energia pela atividade dos músculos do tornozelo, joelho e quadril durante o apoio médio (17). Uma outra possível justificativa para tal diferença entre os MMII de sujeitos com DMI discreta seria a adoção de algum mecanismo compensatório, como já descrito anteriormente na literatura ${ }^{(7,8,11)}$, na tentativa de equilibrar o comprimento dos membros e desenvolver um padrão de marcha simétrico.

Segundo Liu et a/(8), sujeitos com DMI podem adotar uma maior supinação da articulação subtalar, o que resultaria em um pé mais rígido, logo menos apto para amortecer o impacto, e um membro inferior funcionalmente mais longo por aumentar a distância vertical do pé com o solo. Entretanto, espera-se que durante o apoio médio o pé realize a pronação da articulação subtalar com o intuito de absorver o impacto imposto ao membro inferior durante a marcha. Kaufman et al (4) cogitaram ainda que essas adaptações utilizadas na tentativa de igualar funcionalmente a desigualdade podem levar a um aumento de sobrecarga em um dos membros favorecendo o surgimento de sintomas e/ou lesões. Diante disso, os maiores valores de $\mathrm{Fz}_{\min }$ encontrados no $\mathrm{MI}_{\text {menor }}$ dos sujeitos com DMI discretas podem indicar, além de uma menor eficiência mecânica e possível sobrecarga, um mecanismo compensatório adotado por esses sujeitos na tentativa de igualar o comprimento dos membros inferiores e realizar uma marcha simétrica. E uma vez que os valores

\section{REFERÊNCIAS}

1. Brunet ME, Cook SD, Brinker MR, Dickinson JA. A survey of running injuries in 1505 competitive and recreational runners. J Sports Med Phys Fitness. 1990; 30:307-15

2. McCaw ST, Bates BT. Biomechanical implications of mild leg length inequality Br J Sports Med. 1991; 25:10-3

3. Gurney B. Review, Leg length discrepancy. Gait Posture. 2002; 15: 195-206.

4. Kaufman KR, Miller LS, Sutherland D. Gait asymmetry in patients with limb length inequality. J Pediatr Orthop. 1996; 16: 144-50.

5. Hanada E, Kirby RL, Mitchell M, Swuste JM. Measuring leg-length discrepancy by the "iliac crest palpation and book correction" method: reliability and validity. Arch Phys Med Rehabil. 2001; 82: 938-42.

6. Vink P, Huson A. Lumbar back muscle activity during walking with leg inequality. Acta Morphol Neerl Scan. 1987; 25:261-71.

7. Song KM, Halliday SE, Little DG. The effect of limb length discrepancy on gait. J Bone Joint Surg Am. 1997; 79:1690-8.

8. Liu XC, Fabry G, Molenares G, Lammens J, Moens P. Kinematic and kinetic asymmetry in patients with leg length discrepancy. J Orthop Pediatr. 1998; 18:187-9.

9. White SC, Gilchrist LA, Wilk BE. Asymmetric limb loading with true or simulated leg-length differences. Clin Orthop Relat Res. 2004; (421):287-92.

10. Amadio AC, Duarte M. Fundamentos biomecânicos para a análise do movimento humano. São Paulo: Laboratório de Biomecânica/Eefeusp; 1996.

11. D'Amico JC, Dinowitz HD, Polchaninoff M. Limb length discrepancy. An eletro- do Índice de Simetria Absoluto (ISA) para todas as variáveis da FRS vertical e horizontal analisadas no presente estudo, foram muito pequenos ou próximos de zero, pode-se dizer que independente do mecanismo compensatório adotado, os sujeitos do grupo desigualdade apresentaram uma marcha simétrica.

Vale ressaltar que essas compensações podem ter ocorrido em qualquer região ou articulação, no entanto, a não realização de uma avaliação cinemática neste estudo impossibilitou a identificação exata dos mecanismos adotados por esses sujeitos.

Para Brunet et al (1) e Hreljac (20) os desequilíbrios biomecânicos, como a DMI, parecem ser um dos maiores contribuintes para a ocorrência de lesões na corrida. E segundo valores de referência citados por Friberg ${ }^{(14)}$, as desigualdades apresentadas pelos sujeitos de GD já seriam suficientes para causar alterações ortopédicas e de fato, cerca de $50 \%$ dos sujeitos analisados neste estudo já haviam experimentado dor lombar e/ou dor no joelho, e aproximadamente $20 \%$ deles já apresentaram dor no quadril e/ou fratura por stress, muito embora não se podem atribuir diretamente estas alterações às desigualdades apresentadas. Também não se pode afirmar que as diferenças encontradas no presente estudo entre os membros inferiores dos sujeitos com desigualdade discreta, embora em presença de uma marcha simétrica, não tenham representado uma sobrecarga para o aparelho locomotor desses corredores.

Deste modo, por meio da análise das variáveis biomecânicas investigadas neste estudo, pode-se dizer que a velocidade autoselecionada no andar sugerida aos sujeitos avaliados não representou um desafio ao sistema musculoesquelético desses sujeitos, possivelmente por estarem adaptados a maiores sobrecargas uma vez que todos eram corredores de média e longa distância. Além disso, sabe-se que com o aumento da velocidade aumentam-se os valores de FRS, e conseqüentemente, as alterações poderiam ser mais evidentes. Logo, um outro estudo realizando as mesmas análises, mas utilizando maiores velocidades, pode reproduzir de forma mais evidente as possíveis sobrecargas impostas aos sujeitos com desigualdade discreta de membros inferiores.

\section{CONCLUSÃO}

Os achados deste estudo indicam que corredores de média e longa distância com desigualdade estrutural média de membros inferiores da ordem de 1,0 \pm 0,5 $\mathrm{cm}$ para promover uma marcha simétrica, adotam mecanismos compensatórios dinâmicos capazes de gerar sobrecarga adicional ao sistema musculoesquelético.

\section{AGRADECIMENTOS}

Agradecemos à FAPESP (Processo No. 03/08165-7) pelo apoio financeiro concedido. dynographic analysis. J Am Podiat Assoc. 1985; 75: 639-43.

12. Cunha LAM, Pauleto AC, Oliva Filho AL, Moura MFA, Penkal ML. Influência do posicionamento osteoarticular e dos possíveis erros técnicos nos valores obtidos em escanometrias. Rev Bras Ortop. 1996; 31:240-6.

13. Terry Ma, Winell Jj, Green Dw, Schneider R, Peterson M, Marx Rg, et al. Measurement variance in limb length discrepancy: Clinical and radiographic assessment of interobserver and intraobserver variability. J Pediatr Orthop. 2005; 25:197-201

14. Friberg O. Clinical symptoms and biomechanics of lumbar spine and hip joint in leg length inequality. Spine. 1983; 8:643-51.

15. Dahl MT. Limb length discrepancy. Pediatr Clin North Am. 1996; 43: 849 - 65.

16. Nigg BM. Biomechanics of running shoes. Human Kinetics, Champaign, IL; 1986.

17. Winter DA. Biomechanics and motor control of human movement. 2nd ed. New York: John Wiley \& Sons; 1990.

18. Karamanidis K, Arampatzis A, Brüggemann GP. Symmetry and reproducibility of kinematic parameters during various running techniques. Med Sci Sports Exerc. 2003; 35: 1009-16

19. Herzog W, Nigg BM, Read LJ, Olsson E. Asymmetries in ground reaction force patterns in normal human gait. Med Sci Sports Exerc. 1989; 21:110-4.

20. Hreljac A. Impact and overuse injuries in runners. Med Sci Sports Exerc. 2004; 36:845- 9 